\title{
Voltage Unbalance Reduction in Low Voltage Feeders by Dynamic Switching of Residential Customers among Three Phases
}

\author{
Farhad Shahnia, member IEEE, Peter J. Wolfs, Senior member, IEEE, and Arindam Ghosh, Fellow, IEEE
}

\begin{abstract}
To dynamically reduce voltage unbalance (VU) along low voltage distribution feeders, a distributed intelligent residential load transfer scheme is proposed. In this scheme, residential loads are transferred from one phase to another to minimize $\mathrm{VU}$ along the feeder. The central controller, installed at the distribution transformer, observes the power consumption in each house and determines the house(s) to be transferred from an initially connected phase to another. The transfer is carried out by the help of a static transfer switch, with a three-phase input and a single-phase output connection, through which each house is supplied. The steady-state and dynamic performances of the proposed load transfer scheme are investigated by MATLAB analyses and PSCAD/EMTDC simulations.
\end{abstract}

Keywords-Distribution Feeder, Load Transfer, Static Switch, Voltage Unbalance

\section{INTRODUCTION}

In many parts of the world (including Australia, Asia, Europe and Africa), the Low Voltage (LV) residential feeders are usually three-phase, four-wire systems, supplied by Dyn three-phase transformers [1]. Majority of the houses have single-phase power supply (i.e. one of the three phases and neutral) but larger houses may have three-phase connections. Note that this type of system is not common in North America.

Current unbalance and Voltage Unbalance (VU) are one of the main power quality problems in these feeders [2]. VU can be very high in feeders with voltage drops close to the allowable limits, especially if the houses are distributed unequally among the three phases [1]. The growing penetration of rooftop Photovoltaic generators (PV) in these LV feeders has increased the VU problem. The output power of the rooftop PVs is intermittent and the PVs are randomly distributed amongst phases as their installation depends on the customers. Therefore, penetration level, rating and location of PVs in the feeder significantly affect the VU [3]. The growing penetration of plug-in electric vehicles will contribute to further unbalance. In [4], it is shown that they can lead to high VU in both of the charging and discharging modes.

Currently, the utilities minimize the unbalance problem in LV feeders by manually changing the connection phase of some of the costumers to equalize the distribution of the loads

F. Shahnia and A. Ghosh are with Center of Smart Grid and Sustainable Power Systems, Department of Electrical and Computer Engineering, Curtin University, Perth, Australia. P.J. Wolfs is with Power and Energy Center, School of Engineering and Built Environment, University of Central Queensland, Rockhampton, Australia. (farhad.shahnia@curtin.edu.au) amongst the phases [1]. Different methods are proposed for unbalance reduction in LV feeders. In [3], some conventional improvement methods such as feeder cross-section increase or capacitor installation are investigated. In [5], the application of custom power devices such as Distribution Static Compensator (DSTATCOM) is been proposed. It is shown that such devices can balance voltages at their connection terminals. If the terminal voltage is made balanced, the current drawn from the upstream network will be balanced, provided that the supply voltage is balanced. This will prevent the flow of large unbalanced current from the upstream network. In [3], utilization of rooftop PV inverters for exchanging reactive power is proposed for balancing their terminal voltage. Although this is an effective method, it requires PV inverter standards to evolve to accommodate this in future.

In modern distribution networks, sectionalizing switches and normally open tie switches are often used for reconfiguration of the network at Medium Voltage (MV) levels. The main benefits of reconfiguration for the network are reducing the power loss [6], facilitating higher penetration level of distributed generation units [7], improving power quality [8] and faster restoration of service following a fault [9]. In [10], it is shown the network reconfiguration can be carried out by simply changing the phase connection of the three phases in the primary side of the distribution transformer, for VU and power loss reduction. Therefore, based on the known load pattern for each distribution transformer, the optimum phase balancing is carried out. However, this practice is carried out only once and is not dynamic. In [11], it is shown that using Static Transfer Switches (STS), a sensitive load can be supplied from two different feeders by quickly transferring the load from one three-phase feeder to another, to prevent voltage sag/swell affecting it. A similar network reconfiguration and Load Transfer (LT) scheme, derived from [10-11], can be applied in LV feeders to reduce VU in the network.

In this paper, an intelligent dynamic residential LT scheme is proposed. The proposed scheme consists of a central controller, several distributed end-user controllers and STSs. In this scheme, an end-user controller, installed at each house, transmits the power consumption of the house to a central controller, installed at the distribution transformer. The central controller then analyzes the network VU and total power consumption in each phase and determines the house(s) to be transferred from an initially connected phase to another. Commands are then issued to the end-user controller in the 
selected house(s). Upon receiving a command from the central controller, the end-user controller activates the STS to change the phase connection for that house accordingly. The STS has a three-phase input and a single-phase output connection such that any of the three phases can be connected to the single-phase residential load, one at a time. For investigating the performance of the proposed LT scheme, a comprehensive analysis is carried out in MATLAB. Through this, VU, maximum and minimum voltage along a feeder and power mismatch amongst phases of the network under consideration are studied. Following this, the participation levels of the houses in the LT scheme are investigated, considering their locations along the feeder. The algorithm is later modified to utilize a Genetic Algorithm (GA) based optimization to find the optimal phase connections of the houses, to achieve the minimal VU and power mismatch amongst phases. Note that the voltage at each customer bus should be within the specified limits during LTs. This is verified by performing dynamic changes using a power electronics-based STS through simulations in PSCAD/EMTDC.

The main contribution of this paper is in proposing the idea of dynamically transferring the single-phase residential loads from one phase to another, within a three-phase system, to reduce VU along the feeder and power mismatch amongst phases. A practical and readily implementable deadband control algorithm is developed, which reduces LT frequency versus VU. The method can be further refined using any standard optimization method such as GA. A new STS connection strategy is also developed for implementation of the proposal.

\section{Voltage UnBALANCE DEFINITION}

VU in the three-phase electric system is a condition in which the three phase voltages $\left(V_{A}, V_{B}\right.$ and $\left.V_{C}\right)$ differ in amplitude and/or do not have $120^{\circ}$ phase differences between them. There are several methods for calculation and interpretation of VU, as discussed briefly below:

- Phase Voltage Unbalance Rate (PVUR) is defined, based on IEEE Std. 936-1987 [13], as

$$
P V R_{\text {IEEE } 936}=\frac{\operatorname{Max}\left(V_{A}, V_{B}, V_{C}\right)-\operatorname{Min}\left(V_{A}, V_{B}, V_{C}\right)}{\operatorname{Mean}\left(V_{A}, V_{B}, V_{C}\right)}
$$

where Max, Min and Mean are respectively the maximum, minimum and mean functions.

- PVUR is defined, based on IEEE Std. 112-1991 [13], in terms of phase voltages and their average $\left(V_{a v-P}\right)$ as

$$
P V R_{\text {IEEE 112 }}=\frac{\operatorname{Max}\left(\left|V_{A}-V_{a v-P}\right|,\left|V_{B}-V_{a v-P}\right|,\left|V_{C}-V_{a v-P}\right|\right)}{V_{a v-P}}
$$

- Line Voltage Unbalance Rate (LVUR) is defined, based on NEMA std. 1993 [13], in terms of line voltages $\left(V_{A B}, V_{B C}\right.$ and $\left.V_{C A}\right)$ and their average $\left(V_{a v-L}\right)$ as

$$
\begin{gathered}
L V U R_{N E M A}=\frac{\operatorname{Max}\left(\left|V_{A B}-V_{a v-L}\right|,\left|V_{B C}-V_{a v-L}\right|,\left|V_{C A}-V_{a v-L}\right|\right)}{V_{a v-L}} \quad \text { (3) } \quad S_{\alpha}^{V U \%}=\frac{\alpha}{\mathrm{VU} \%} \cdot \frac{\partial}{\partial \alpha}(V U \%) \\
V U \%=\sqrt{\frac{2\left(V_{A}^{2}+V_{B}^{2}+V_{C}^{2}\right)+2 V_{A} \cdot V_{B} \cos \left(\theta_{A B}+60^{\circ}\right)+2 V_{B} V_{C} \cos \left(\theta_{B C}+60^{\circ}\right)+2 V_{C} V_{A} \cos \left(\theta_{C A}+60^{\circ}\right)}{\left(V_{A}^{2}+V_{B}^{2}+V_{C}^{2}\right)+2 V_{A} \cdot V_{B} \cos \left(\theta_{A B}-120^{\circ}\right)+2 V_{B} V_{C} \cos \left(\theta_{B C}-120^{\circ}\right)+2 V_{C} V_{A} \cos \left(\theta_{C A}-120^{\circ}\right)}}
\end{gathered}
$$

- CIGRE report 1986 [12] defines VU in terms of line voltages as

$$
\begin{aligned}
& V U_{C I G R E}=\sqrt{\frac{1-\sqrt{3-6 \beta}}{1+\sqrt{3-6 \beta}}} \\
& \text { where } \beta=\frac{\left|V_{A B}\right|^{4}+\left|V_{B C}\right|^{4}+\left|V_{C A}\right|^{4}}{\left.\left.|| V_{A B}\right|^{2}+\left|V_{B C}\right|^{2}+\left|V_{C A}\right|^{2}\right)^{2}}
\end{aligned}
$$

- Voltage Unbalance True Definition $\left(V U_{T D}\right)$ is defined, based on IEEE Std. 1996 [13] and IEC Std. 61000-4-27, as

$V U_{T D}=\left|\frac{V_{-}}{V_{+}}\right|=\left|\frac{V_{A B}+a^{2} V_{B C}+a V_{C A}}{V_{A B}+a V_{B C}+a^{2} V_{C A}}\right|$

where $V_{\text {- }}$ and $V_{+}$are respectively the negative and positive sequence of the voltage and $a=1 \angle 120^{\circ}$.

- Voltage Unbalance in four-wire systems $\left(V U_{4 \text {-wire }}\right)$ is defined in [13] as

$V U_{4-\text { wire }}=\frac{\sqrt{V_{-}^{2}+V_{0}^{2}}}{\left|V_{+}\right|}$

to consider the zero sequence of voltage $\left(V_{0}\right)$ in unbalance calculations, which is not considered in (5). It is stated in [13] that in four-wire systems, zero sequence voltage often makes a larger contribution to phase voltage magnitude imbalance than negative sequence components. This is referred to as percentage VU (VU\%) in the rest of the paper.

For a three-phase, four-wire LV feeder with equally sized phase and neutral conductors, it can be shown that $L V U R_{N E M A}$ and $V U_{T D}$ are equivalent, while $V U_{4 \text {-wire }}$ is higher than $V U_{T D}$ but lower than PVUR $R_{\text {IEEE936 }}$ and PVUR $R_{\text {IEEE1 12. }}$.

According to [14], the allowable limit for $\mathrm{VU}$ is $2 \%$ in $\mathrm{LV}$ and MV networks. UK Engineering Recommendation P29 not only limits VU to $2 \%$, but also limits it to $1.3 \%$ at the load point [15]. ANSI standard for "Electric Power Systems and Equipment Voltage Ratings $(60$ Hertz)" recommends that electrical supply systems should be designed to limit the maximum VU to $3 \%$ when measured at the electric utility end points at no-load conditions [16].

VU at, a particular node, can be minimized by equalizing the loads in each phase at that node. Two analytical methods can be used for defining the load change for VU reduction, as discussed below:

\section{A. Sensitivity Analysis}

Based on the symmetrical components method for phase voltages, (6) can be expressed as (7) where $\theta_{A B}=\theta_{A}-\theta_{B}$, $\theta_{B C}=\theta_{B}-\theta_{C}$ and $\theta_{C A}=\theta_{C}-\theta_{A}$. VU Sensitivity versus $\alpha$, where $\alpha$ is any of $V_{A}, V_{B}, V_{C}, \theta_{A}, \theta_{B}$ and $\theta_{C}$ parameters, is calculated as [17] 
When calculating VU sensitivity versus any of these parameters, the other parameters need to be constant. This is based on the assumption that they are independent of each other. In this case, VU sensitivity analysis determines the $\alpha$ parameter that has the most effect on VU reduction. Then, a proper load change should be selected to reflect the desired variation in the determined $\alpha$ parameter.

\section{B. Vector Analysis}

Consider a balanced three-phase four-wire source with a single-phase load. The load is assumed to draw a constant current $I$ at power factor of $\cos \varphi$ lagging. If the load is connected to Phase-A, the current in this phase is $i_{A}=I \angle-\varphi$ and the sequence components of the current are

$I_{+}=I_{-}=I_{0}=\frac{I}{3} \angle-\varphi$

If the load is connected to Phase- $\mathrm{B}$, the current in this phase is $i_{B}=I \angle-120^{\circ}-\varphi$ and the current sequence components are

$$
I_{+}=\frac{I}{3} \angle-\varphi \quad I_{-}=\frac{I}{3} \angle+120^{\circ}-\varphi \quad I_{0}=\frac{I}{3} \angle-120^{\circ}-\varphi
$$

If the load is connected to Phase- $\mathrm{C}$, the current in this phase is $i_{C}=I \angle+120^{\circ}-\varphi$ and the current sequence components are

$$
I_{+}=\frac{I}{3} \angle-\varphi \quad I_{-}=\frac{I}{3} \angle-120^{\circ}-\varphi \quad I_{0}=\frac{I}{3} \angle+120^{\circ}-\varphi
$$

Now, for studying the effects of a LT from one phase to another, let the load in Phase-A be transferred to Phase-B. In this case, the changes in the current sequence components are

$$
\begin{aligned}
& \Delta I_{+}^{A \rightarrow B}=-\frac{I}{3} \angle-\varphi+\frac{I}{3} \angle-\varphi=0 \\
& \Delta I_{-}^{A \rightarrow B}=-\frac{I}{3} \angle-\varphi+\frac{I}{3} \angle+120^{\circ}-\varphi=\frac{I}{\sqrt{3}} \angle+150^{\circ}-\varphi \\
& \Delta I_{0}^{A \rightarrow B}=-\frac{I}{3} \angle-\varphi+\frac{I}{3} \angle-120^{\circ}-\varphi=\frac{I}{\sqrt{3}} \angle-150^{\circ}-\varphi
\end{aligned}
$$

Similarly, the changes in the current sequence, when the load is transferred from Phase- $\mathrm{B}$ to $\mathrm{C}$ and from Phase-C to A, are

$$
\underset{B \rightarrow C}{\left[\begin{array}{c}
\Delta I_{+} \\
\Delta I_{-} \\
\Delta I_{0}
\end{array}\right]}=\frac{I \angle-\varphi}{\sqrt{3}}\left[\begin{array}{c}
0 \\
1 \angle-90^{\circ} \\
1 \angle+90^{\circ}
\end{array}\right] \text { and } \underset{C \rightarrow A}{\left[\begin{array}{c}
\Delta I_{+} \\
\Delta I_{-} \\
\Delta I_{0}
\end{array}\right]}=\frac{I \angle-\varphi}{\sqrt{3}}\left[\begin{array}{c}
0 \\
1 \angle+30^{\circ} \\
1 \angle-30^{\circ}
\end{array}\right]
$$

From (9)-(10), it can be seen that, when the load is transferred from one phase to another, the change in the positive sequence of current is zero. However, the negative and zero sequences of the current change.

In a similar way, it can be proved that the LT from Phase-B to A results in $180^{\circ}$ phase change compared to LT from Phase-A to $\mathrm{B}$, for the negative and zero sequences. It is also similar for transfers from Phase-C to B and from Phase-A to $\mathrm{C}$. The change of the negative and zero sequence components of the current, for a LT between any two phases, is shown in Fig. 1.

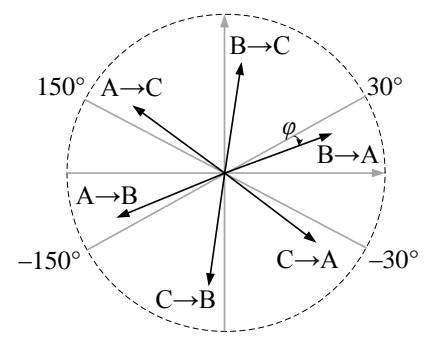

(a)

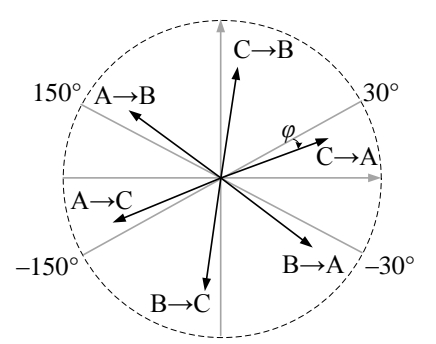

(b)
Fig. 1. (a) Change in current zero sequence for LT between two phases, (b) Change in current negative sequence for $\mathrm{LT}$ between two phases.

\section{LOAD TRANSFER SCHEME}

Fig. 2(a) shows the schematic of a typical radial distribution network in a suburban area in Australia. The MV feeder supplies several three-phase distribution transformers and each distribution transformer supplies a few tens of residential houses with single-phase connections.

The main objective of the proposed LT scheme is to ensure that $\mathrm{VU}$ is reduced along the feeder while the power mismatch amongst the phases in the secondary side of the distribution transformer is also reduced. The preliminary stage of this scheme is that the utilities are aware of the phase connection of each house. If this is not known, the method presented in [18] can be utilized to define the connections.

The main assumptions for the deployment of the proposed LT scheme are availability of:

- Distributed smart operating devices

- Communication coverage

- Centralized controller

and the main requirements of the scheme are:

- Effectiveness: The scheme should reduce the VU along the feeder and the power mismatch amongst the phases.

- Low cost: The hardware cost should be minimal.

- Scalability: The scheme should be easily scalable to larger networks.

- Robustness: The scheme should be fault tolerant.

Detailed descriptions of the proposed scheme and required equipment, controllers and communication are given below.

\section{A. Smart Meters}

The LT scheme requires access to instantaneous power consumption of the residential loads. For this, all the houses participating in the LT scheme are equipped with smart meters [19] that transmit the power consumption of the house to the central controller in 15-min intervals.

\section{B. Controllers}

In the proposed scheme, there are two types of controllers. A central controller is installed at the distribution transformer and analyzes the network VU and power mismatch between three phases after receiving the power consumption data from the smart meters. Then, it chooses the candidate house(s) in which LT is required and subsequently sends a control command to the selected house(s).

An end-user controller is installed at each LT participating house. This controller activates the STS once it receives a control command from the central controller. The end-user 
controllers in one feeder only correspond with the central controller of that feeder.

\section{Communication}

Different communication methods, such as power line carriers, optical fiber Ethernet, internet, $3 \mathrm{G} / 4 \mathrm{G}$ wireless, WiFi and ZigBee, have been already utilized in electric distribution and transmission networks [20]. However, in recent years, ZigBee has become the most preferred communication method for data transfer in smart grid distribution network applications. Therefore, in this paper, ZigBee-based communication is assumed for transferring the control commands from the central controller to the end-user controllers. Similarly, the end-user controllers send the confirmation of successful LT to the central controller.

Two important parameters for the communication are the coverage area and data transfer rate. In this paper, the length of the LV distribution feeders is assumed to be about $400 \mathrm{~m}$ long, which is normal in Australia. Therefore, two outgoing feeders from a distribution transformer, spread in two opposite directions, can cover an area of $800 \mathrm{~m}$. The LT is proposed to be carried out on 15-min intervals. The available ZigBee devices along with their range extenders can cover an area of $1.6 \mathrm{~km}$ and have a data rate up to $250 \mathrm{kHz}$ [21]. However, for this application, a very low bandwidth is sufficient.

\section{Static Transfer Switches}

The residential load is transferred from one phase to another through an AC Static Transfer Switch, as shown in Fig. 2(b). The STS is composed of three switching devices, one for each phase. Each switching device is composed of a Triac or anti-parallel thyristors, with overvoltage protection and snubber circuits connected in parallel [2]. Each switching device is connected to one of the three phases of the feeder in input and their outputs are connected together and to the load. Only one switching device is operating at a time, to prevent short-circuiting the phases. No auxiliary commutation circuit is used in the proposed STS connection strategy.

Assume that switch-1 is on and the load is connected to Phase-A. Once the LT command is received by the end-user controller to transfer the load from Phase-A to B, it blocks the gate signals for switch-1. However, switch-1 still continues to supply the load, until the current falls below its holding current. Subsequently, it turns off and the gate signals are applied to switch-2. This lack of timing control for a Triac/thyristor is the main drawback of the proposed STS. Gate Turn-Off Thyristors (GTO) or Insulated Gate Bipolar Transistors (IGBT) can also be utilized; however, they required complex driver circuits, are more expensive and have higher conduction losses. These are the main reasons for choosing Triacs/thyristors for the STS.

\section{E. Reliability Issues}

There are two main reliability concerns with the proposed scheme. The first relates to the failure probability (or lost data packets) in the central controller or ZigBee-based communication system [22]. The second one relates to the failure of STS including controller, semiconductors, PCB, etc. [23].

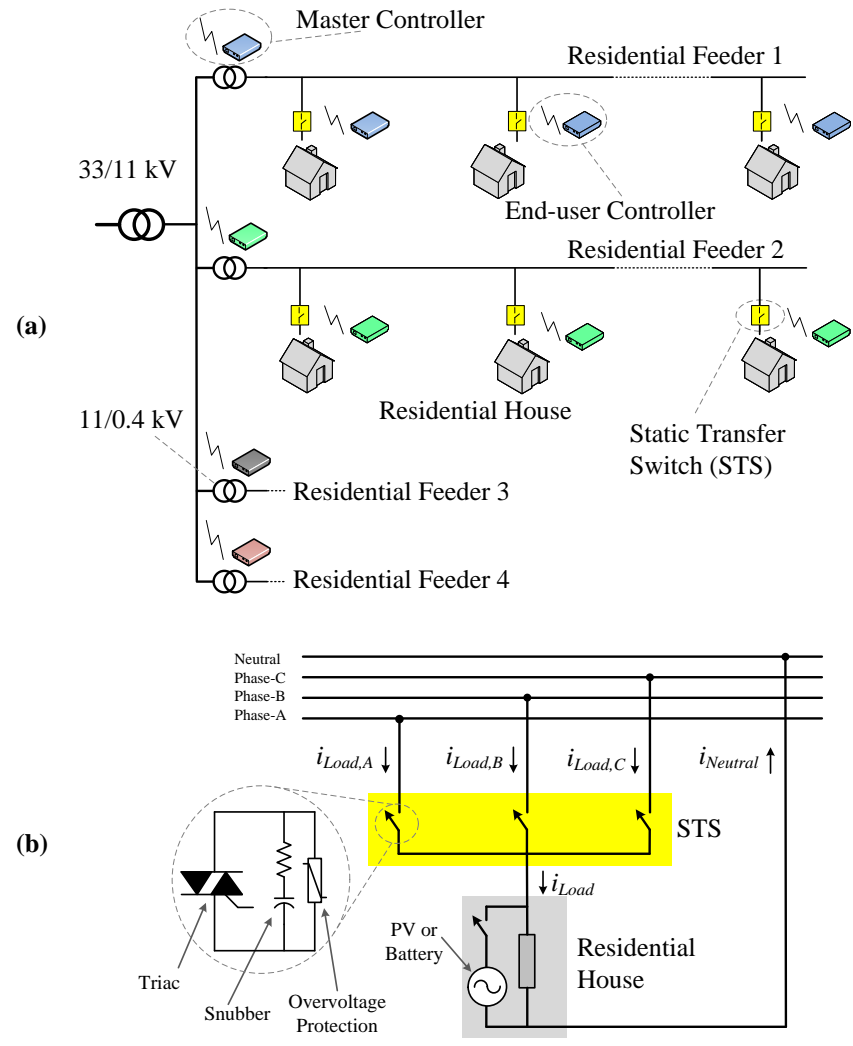

Fig. 2. (a) Schematic of the proposed LT scheme in LV feeders, (b) Schematic of the proposed STS for residential customers.

A failure in the central controller and communication system stops the LT scheme and the system remains in the current state. The houses receive an uninterrupted supply but VU may rise with the time.

Failure in STS can be considered separately for failure in an open-circuit or a short-circuit state. If the STS fails in open-circuit, then the house is disconnected and the STS should be bypassed until it is replaced. However, if the STS fails in short- circuit, the house is no longer able to participate in the LT scheme but it receives an uninterrupted supply.

\section{F. Load Transition Dynamic Issues}

Transferring a load from one phase to another results in a dynamic input voltage variation for that load. Voltage disturbance limit curves, such as the ITI curve (formerly CBEMA), define the acceptable regions of the voltage variations for electrical appliances. Manufacturers design their products to tolerate the disturbances defined by these curves [24-25]. If the voltage variations during LT transitions are kept within the acceptable region of the ITI curve, there will be no adverse effects for the residential electrical.

The frequency of LT is limited by the selection of 15-min intervals between two consequent switching events. In addition, as discussed in the next section, a deadband controller is also developed that activates the LT scheme only when the VU is above an acceptable (desired) limit. The deadband controller restricts the LTs to the periods with unacceptable VU in the network and hence, the number of LTs reduces signifi- 
cantly. Discrete LT and the deadband controller minimize the LT number and its peripheral effects on voltage variations.

\section{G. Cost Concerns}

Smart meters provide a point of reference. The approximate cost of a smart meter is $\$ 100$, based on the reports of the recent large scale deployment of smart meters [26]. The load switching device is of comparable complexity and likely to be of the same cost. With the cost of a 25 A Triac in the range of a few dollars, the total cost of an STS including the end-user controller will not exceed $\$ 100$. Given the fact that many utilities worldwide are stipulating the use of smart meters, the only added cost is that of the STS.

\section{LOAD TRANSFER CONTROL AlgORITHM}

A three-phase four-wire LV feeder is assumed to have several single-phase loads, distributed unequally among the phases. It is necessary to determine the preferred LTs that minimize VU.

A LT in any bus will result in the voltage magnitude and angle change in all phases and all buses. Therefore, the assumption of independency between all the three phase voltage magnitudes and angles is invalid. Hence, the VU sensitivity analysis, discussed in Section II(A), cannot be used.

Equations (9)-(10) are based on the assumption of a balanced voltage source. However, in reality, it is quite possible that the voltages at any bus are already unbalanced due to other loads connected to the upstream side of the feeder. In addition, the problem is even more complicated by the diversities and unequal distributions of the loads, along the feeder. Therefore, the vector analysis, discussed in Section II(B), is also not suitable.

Due to limitations of the sensitivity analysis and the vector analysis for VU minimization in a feeder, a method is developed in this paper which is not computation intensive, is very straight forward and easy to be implemented. This method is discussed below:

\section{A. Highly-loaded to Low-loaded LT with Deadband Control}

$\mathrm{VU}$ at each bus is proportional to the difference between the voltage magnitudes of the three phases in that bus. Therefore, to reduce $\mathrm{VU}$ in each bus, a method that equalizes the amplitude of all three phase voltages is implemented. This is achieved by transferring the load from the highly-loaded phase to the low-loaded phase at that bus. An exhaustive method is used for applying a load change from highlyloaded phase to low-loaded phase in each bus followed by calculation of VU for all buses. This process is continued until the least VU for all buses along the feeder is achieved. The variations in VU are monitored to prevent VU increase due to an inappropriate LT. The LT which results in the least VU for all buses in the network is chosen as the desired LT. The flowchart of the control algorithm is shown in Fig. 3(a).

The main aim of this method is VU reduction in the network; however, it indirectly improves the voltage variations and the power mismatch amongst the phases. This method does not result in the globally optimum condition; however, its main advantage is that only a few LTs are required.

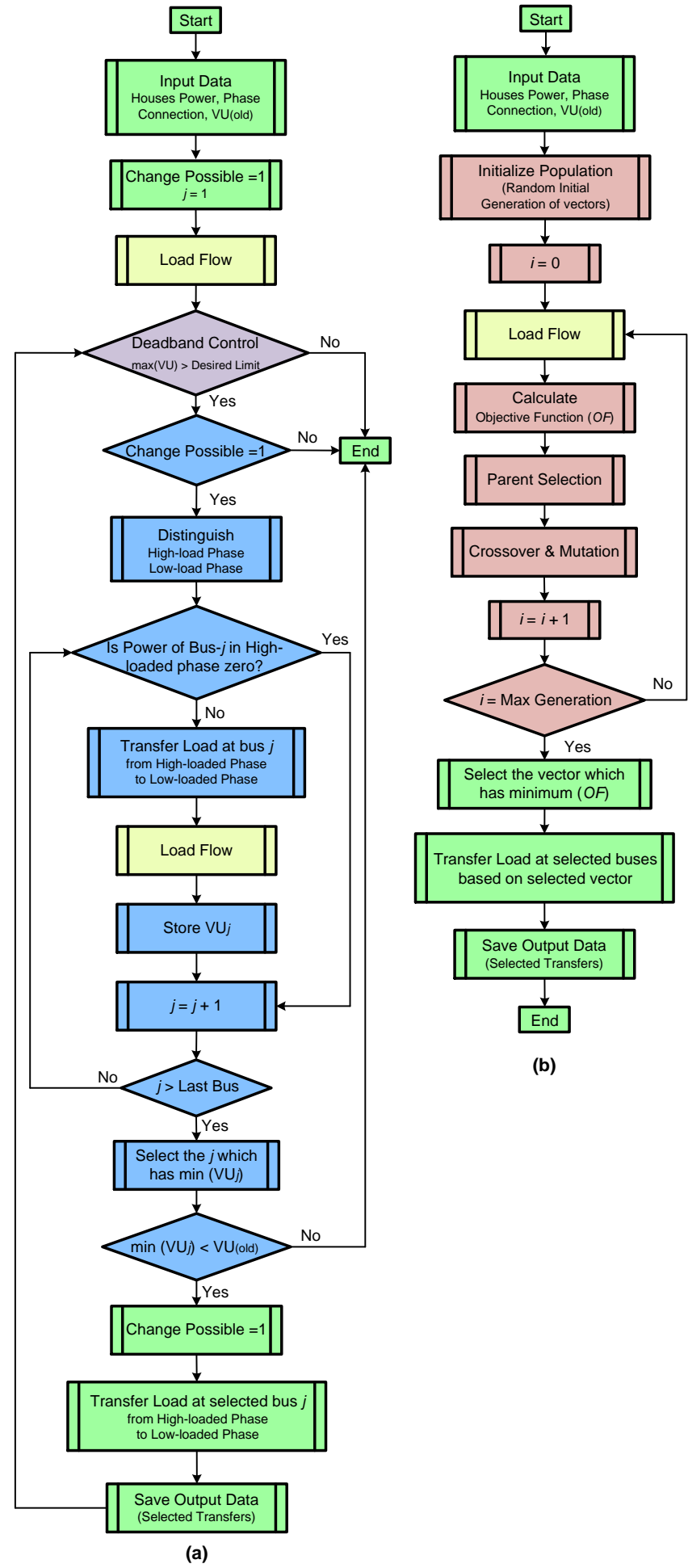

Fig. 3 (a) LT flowchart based on highly-loaded to low-loaded with deadband control, (b) LT flowchart based on GA optimization.

\section{B. Genetic Algorithm-Based LT}

$\mathrm{VU}$ can be reduced even further if a general optimization is carried. Any optimization tool can be used for this purpose. In this paper, a GA-based method is chosen. This method is based on assuming a vector with column number equal to the number of the houses in the network. The value of each vec- 
tor array (cell) can be 1, 2 or 3 which represents respectively Phase-A, B and C connection for each house. Each cell must have a value between 1 and 3, at any time, to indicate that it is connected to one phase.

The implemented genetic algorithm is a commonly used one [27] and hence is not discussed here. A population size of 100 is used. The method has a one-point crossover with a randomly selected truncation point and a constant probability of $30 \%$. Similarly, a one-point mutation with a randomly selected truncation point and a constant probability of $5 \%$ is used. The considered objective function is

$O F_{p}=\beta_{1} \cdot \max \left(V U_{p}\right)+\beta_{2} \cdot \max \left(\Delta P_{p}\right)$

where $O F_{p}$ is the objective function calculated for each population $p, V U_{p}$ and $\Delta P_{p}$ are respectively the vector of $\mathrm{VU}$ along the feeder and power mismatch amongst the phases for each population while $\beta_{1}$ and $\beta_{2}$ are respectively the penalty factors for VU and power mismatch. The GA parameters and the penalty factors need to be tuned to result in fast convergence and lower $O F$ in the network under consideration. Fig. 4(b) shows the flowchart of the GA-based LT control algorithm.

The main advantage of an optimization-based method is finding the most suitable phase connection for the houses in each interval so that the $O F$ is minimized. However, this results in a high number of LTs in each switching event.

\section{SimUlation RESUltS}

In this section, a few MATLAB-based steady-state analyses are presented, followed by dynamic simulation results using PSCAD/EMTDC. Only one residential feeder of Fig. 3(a) is considered. The residential feeder is assumed to be supplied by a three-phase, $100 \mathrm{kVA}, 11 / 0.4 \mathrm{kV}, 50 \mathrm{~Hz}$, Dyn1 distribution transformer, with per-unit impedance of $4 \%$. The LV feeder is an aerial, three-phase, four-wire system which is composed of 4 similar Mercury conductors (i.e. 7/4.5 AAC with $Z=0.315+j 0.259 \Omega / \mathrm{km}$ [28]). The overhead line has a length of $400 \mathrm{~m}$, distributed over $120 \mathrm{~cm}$ cross-arms, with ABCN horizontal configuration [29]. This feeder supplies 30 houses through 10 poles (buses) with equal separations (i.e. $40 \mathrm{~m}$ ) along the LV feeder (i.e. 1 house per phase per pole).

The utilized residential load profiles in this analysis, shown in Fig. 4 (a), are based on the data from smart meters, installed in a suburban area in Perth, Australia, that are received in 15-min intervals [30]. From this figure, it can be seen that some loads have negative load profile at some periods which is due to their installed PV units. The loads are assumed to be constant PQ loads for this study.

For steady-state analyses, the main program is comprised of two sub-programs - the intelligent LT program and a load flow program, as shown in Fig. 3(a) and (b). In this study, an unbalanced load flow analysis based on backward/forward sweep concept is developed for the radial three-phase fourwire system [31]. The effectiveness of the proposed LT scheme is studied through steady-state case studies. A few of these are described below. (a)

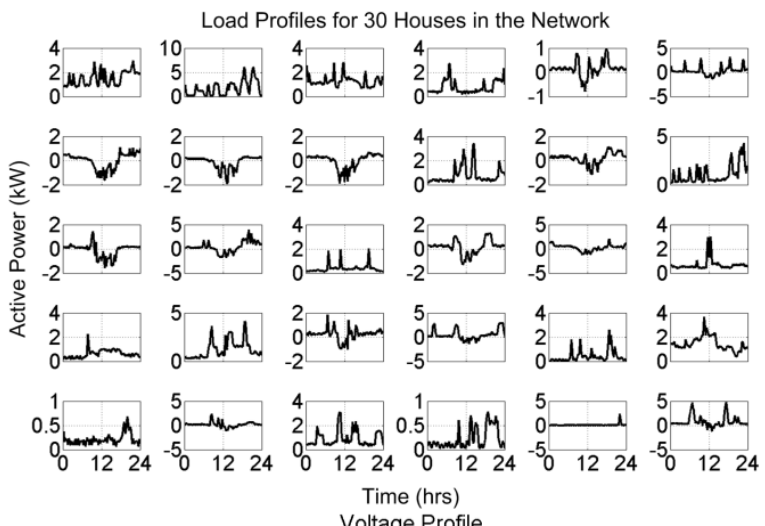

(b)
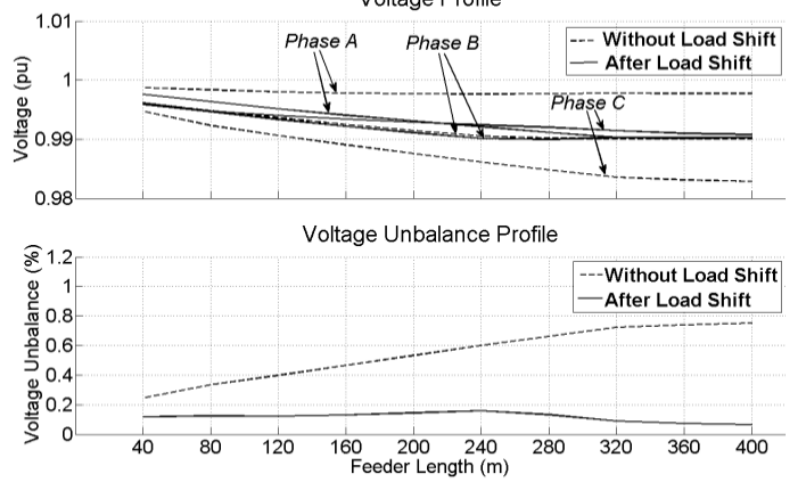

Fig. 4.(a) Load profile for the participating 30 houses in the LT scheme,

(b) Network voltage profile before $\&$ after LT scheme, (c) Network VU profile before \& after LT scheme.

\section{A. Highly-loaded to Low-loaded LT Control}

The LT scheme is implemented as described in Section III along with the control algorithms proposed in Section IV. The deadband controller is assumed to be inactive. This implies that the LT occurs irrespective of the VU level.

The three-phase voltage profile of the feeder before and after 3 LTs, in one switching event, is shown in Fig. 4(b). The VU profile is also shown in Fig. 4(c). As seen in Fig. 4(b), after $3 \mathrm{LTs}$, the difference in the voltage profiles of all the three phases reduces significantly; hence, the VU in the feeder is reduced enormously, as shown in Fig. 4(c). Table 1 lists the numerical results for $\mathrm{VU}$ in the feeder, for the initial case and after each LT, as well as the data of the selected LTs.

Now, the analysis is carried out for a $24-\mathrm{hr}$ period, using the residential load profile data of Fig. 4(a). Maximum of VU along the feeder is shown in Fig. 5(a), before and after applying the proposed LT scheme. This figure shows that the LT scheme is highly successful in reducing the VU along the feeder. The maximum of network VU was $2.23 \%$ which is reduced to $0.16 \%$ after LTs. The maximum VU experienced in the considered 24-hr period, after LTs, is $0.77 \%$.

The minimum voltage along the feeder before and after applying the proposed LT scheme is shown in Fig. 5(b). It can be seen that the minimum voltage in the feeder is improved by the proposed LT scheme. As an example, the minimum voltage in the feeder was $0.97 \mathrm{pu}$ which is increased to 0.99 pu, after LTs.

The maximum voltage in the feeder before and after applying the proposed LT scheme is shown in Fig. 5(c). It can be 
seen that the maximum voltage in the feeder is reduced after LTs. It is to be noted that these changes are the consequence of VU reduction and are not controlled directly.

It is highly desirable to achieve better results with fewer LTs in each switching event. Fig. 5(d) shows that the total number of LTs/switching event is between 1 and 9. This shows that maximum of $30 \%$ of the houses were active in any switching event.

Fig. 5(e) shows the total number of LTs/house at each bus of the network. It can be seen that all houses were active in the LT scheme but some had more transfers than others. In Fig. 5(f), the phase connection of each house is shown during the 24-hr period. This figure shows how quickly the phase connection of a house is changed by the LT scheme.

To analyze if there might be any specific time per day that the number of LTs are higher, the above study is extended for a 7-day period, using the available real load profile data. Fig. $5(\mathrm{~g})$ shows the total number of LTs/hour, when higher than 20 , for this period. It can be seen that the number of LTs is high between 7 and 10 AM and between 4 and 6 PM, every day (i.e. the first and second demand peak of the feeder, in the day).

The total number of LTs/hour and LTs/switching event is given, for the 7-day period, in Table. 2. From this table, it can be seen that for every switching event, on an average, a maximum of $30 \%$ of the houses (i.e. 10 houses) and a minimum of $3 \%$ of the houses (i.e. 1 house) are transferred. It can also be seen that the maximum number of LTs/hour is about 3 times of its minimum number. In addition, in this period, on an average, a minimum number of 2 and a maximum number of 13 LTs/house are observed.

\section{B. Highly-loaded to Low-loaded LT with Deadband Control}

As discussed in Section IV(A), to reduce the number of LTs, a deadband controller is designed that activates the LT scheme only when the VU is higher than the acceptable level. The network of Case-A is considered again where the deadband controller is active for three separate cases - for VU levels of $0.5 \%, 1 \%$ and $1.5 \%$. The results of applying the highly-loaded to low-loaded LT control algorithm are shown in Fig. 6, for each VU level separately. It can be seen that as the acceptable level of VU is increased from $0.5 \%$ to $1.5 \%$, the number of LTs is decreased significantly. For example, by reducing the acceptable level of VU from $1.5 \%$ to $0.5 \%$, the total number of LTs, in a 24-hr period, is increased from 52 to 254 (i.e. 5 times).

\section{Genetic Algorithm-Based LT}

Another study is carried out to investigate the performance of GA-based LT scheme, discussed in Section IV(B). The results of this study for the network of Case-A are shown in Fig. 7.The results are given for the network after applying the LT scheme based on two different optimizations. In the first case, only VU is minimized (i.e. $\beta_{1}=1 \& \beta_{2}=0$ ); while in the second one, both of VU and power mismatch amongst the phases are minimized (i.e. $\beta_{1}=1 \& \beta_{2}=20$ ). It is to be noted that the above penalty factors are tuned to achieve lower $O F$ for a crossover probability of $30 \%$ and mutation probability of $5 \%$, in the network under consideration.

Fig. 7 shows that the VU observed in the network after applying the GA-based optimization is less than the one after applying the highly-loaded to low-loaded LT algorithm. However, this leads to a larger number of LTs (i.e. 160\%). In addition, this figure shows that the power mismatch amongst the phases is reduced significantly in GA-based LT scheme, when this parameter is considered in the $O F$.

\section{Dynamic Simulation Results}

As discussed in Section III $(\mathrm{F})$, the dynamic voltage variations for the residential customers during LT should be within the acceptable region of the ITI curve. For studying the dynamic performance of the STS, the system shown in Fig. 2(b) is modelled in PSCAD/EMTDC, in detail. It is assumed that a single-phase $2 \mathrm{~kW}$ load with power factor of 0.95 lagging is supplied by a three-phase $240 \mathrm{~V}$ RMS voltage through an STS, as described in Section III(D). First, it is assumed the load is being supplied from Phase-A. At $t=0.5 \mathrm{~s}$, a command is received from the central controller to the end-user controller to transfer the load to Phase-B followed by another command at $t=1 \mathrm{~s}$ to transfer the load to Phase-C.

The instantaneous voltage and current waveforms of the load are shown in Fig. 8(a) and (b), while their RMS are shown in Fig. 8(c) and (d). Note that, the current waveform is scaled up for better clarity.

Table 1. VU in each bus along the feeder before and after each LT in highly-loaded to low-loaded LT control algorithm.

\begin{tabular}{|c|c|c|c|c|c|c|c|c|c|c|c|}
\hline Bus number & & 1 & 2 & 3 & 4 & 5 & 6 & 7 & 8 & 9 & 10 \\
\hline Without LT & & 0.24 & 0.33 & 0.39 & 0.46 & 0.53 & 0.60 & 0.66 & 0.72 & 0.74 & 0.75 \\
\hline After $1^{\text {st }} \mathrm{LT}$ & LT at Bus- 8 Phase $\mathrm{C} \rightarrow \mathrm{A}$ & 0.09 & 0.11 & 0.10 & 0.10 & 0.11 & 0.12 & 0.16 & 0.22 & 0.23 & 0.24 \\
\hline After $2^{\text {nd }}$ LT & LT at Bus-7 Phase $\mathrm{C} \rightarrow \mathrm{B}$ & 0.07 & 0.06 & 0.03 & 0.03 & 0.06 & 0.09 & 0.13 & 0.17 & 0.17 & 0.17 \\
\hline After $3^{\text {rd }} \mathrm{LT}$ & LT at Bus-6 Phase $\mathrm{A} \rightarrow \mathrm{B}$ & 0.10 & 0.09 & 0.09 & 0.10 & 0.12 & 0.14 & 0.14 & 0.12 & 0.10 & 0.09 \\
\hline
\end{tabular}

Table 2. LT analysis data based on highly-loaded to low-loaded control algorithm in a 7-day period.

\begin{tabular}{|c|c|c|c|c|c|c|c|c|}
\hline Day & & 1 & 2 & 3 & 4 & 5 & 6 & 7 \\
\hline \multirow{2}{*}{ Number of LTs/switching event } & Maximum number & 9 & 9 & 10 & 10 & 9 & 9 & 9 \\
\hline & Minimum number & 1 & 2 & 1 & 2 & 2 & 1 & 2 \\
\hline \multirow{2}{*}{ Number of LTs/hour } & Maximum number & 33 & 29 & 30 & 20 & 29 & 29 & 28 \\
\hline & Minimum number & 8 & 11 & 13 & 13 & 10 & 12 & 13 \\
\hline \multirow{2}{*}{ Number of LTs/house } & Maximum number & 12 & 12 & 12 & 15 & 12 & 14 & 13 \\
\hline & Minimum number & 1 & 3 & 2 & 3 & 1 & 2 & 2 \\
\hline
\end{tabular}




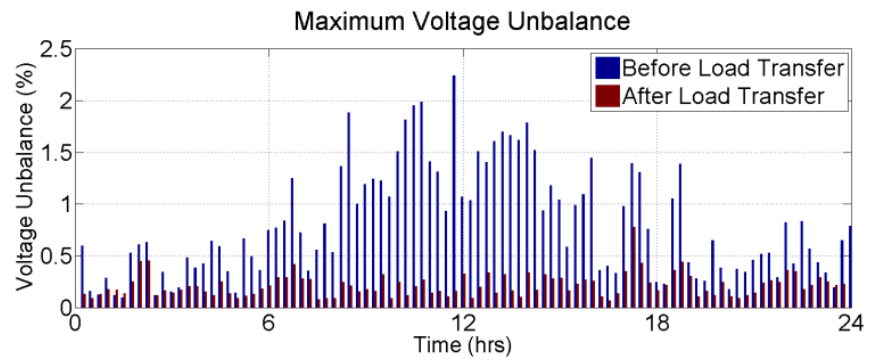

(a)

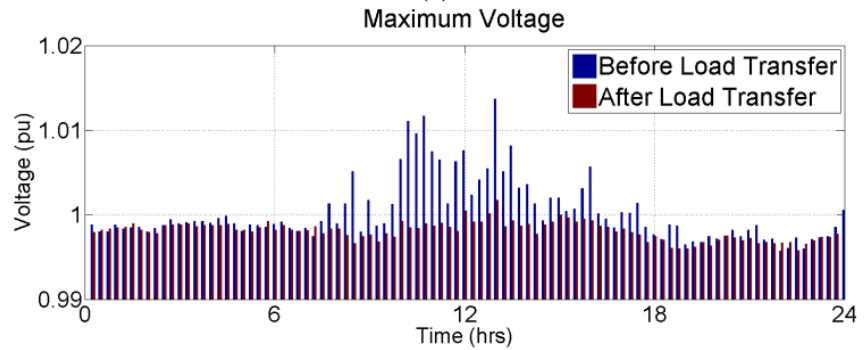

(c)

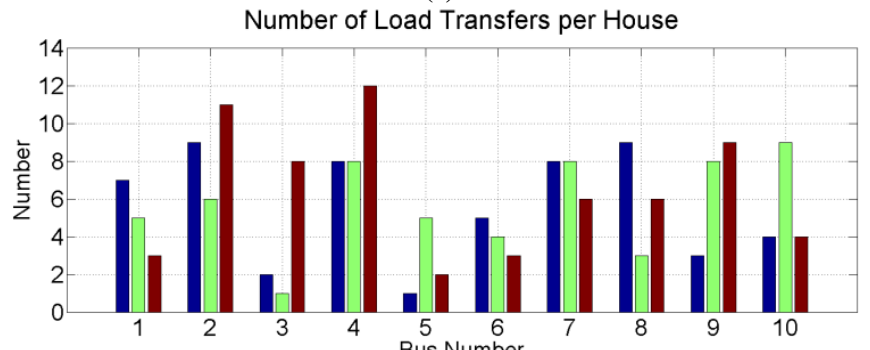

(e)

Fig. 5. Analysis results for the LT scheme based on highly-loaded to low-loaded control algorithm without deadband control:

(a) Maximum VU in the feeder before \& after LTs,

(b) Minimum voltage magnitude in the feeder before $\&$ after LTs,

(c) Maximum voltage magnitude in the feeder before \& after LTs,

(d) Total number of LTs/switching event,

(e) Total number of LTs/house,

(f) Demonstration of phase connection of each house in the network,

(g) Total number of LTs/hour in a 7-day period.
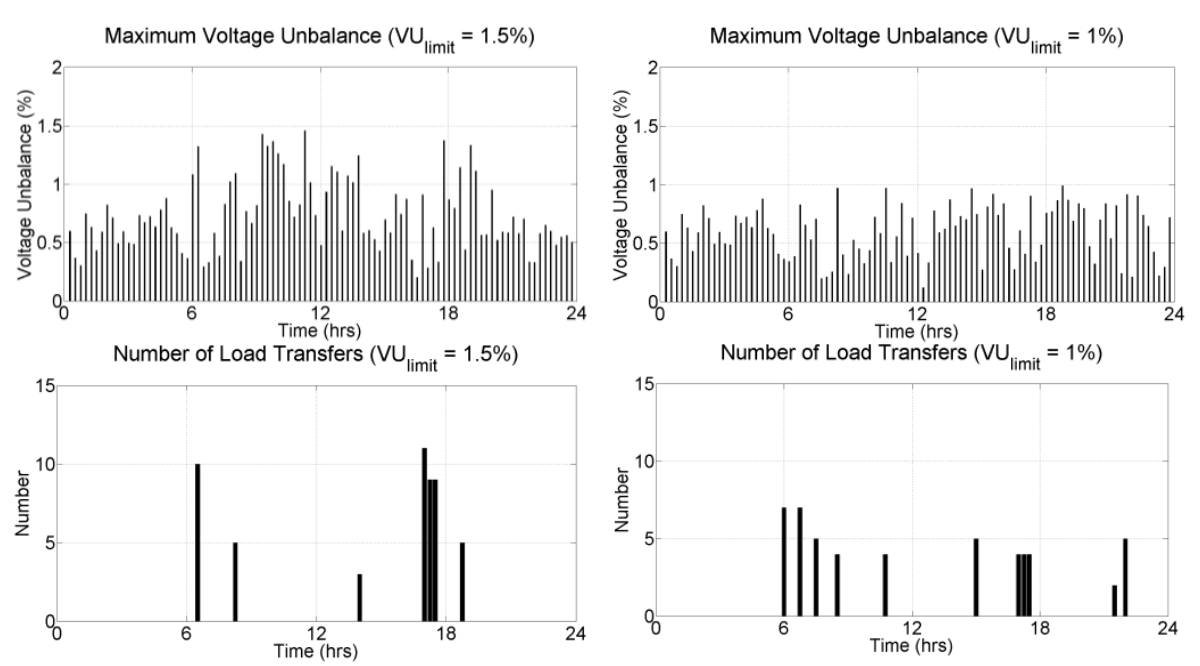

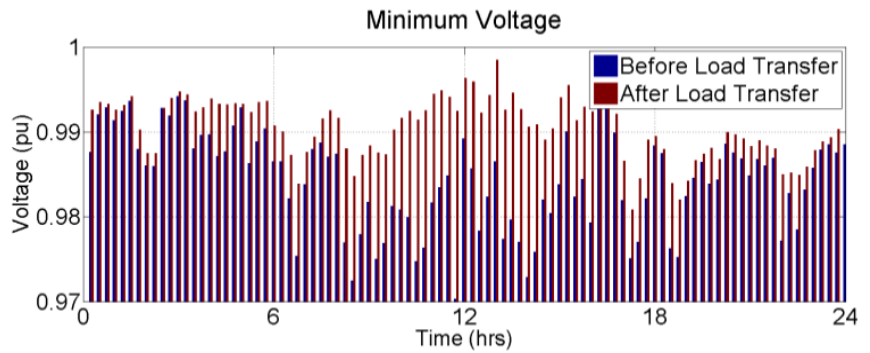

(b)

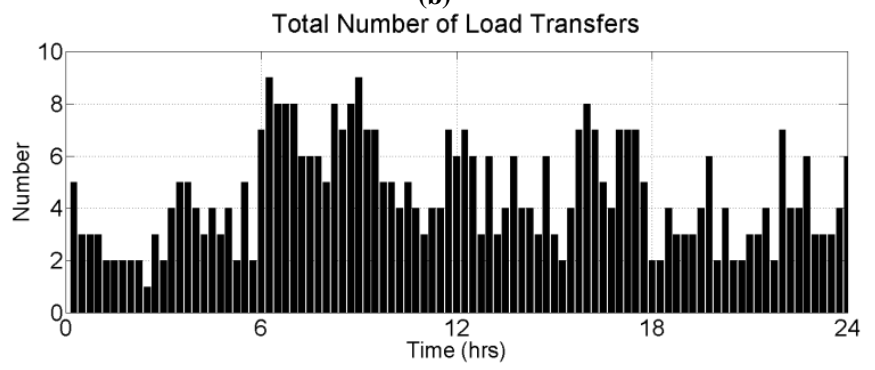

(d)

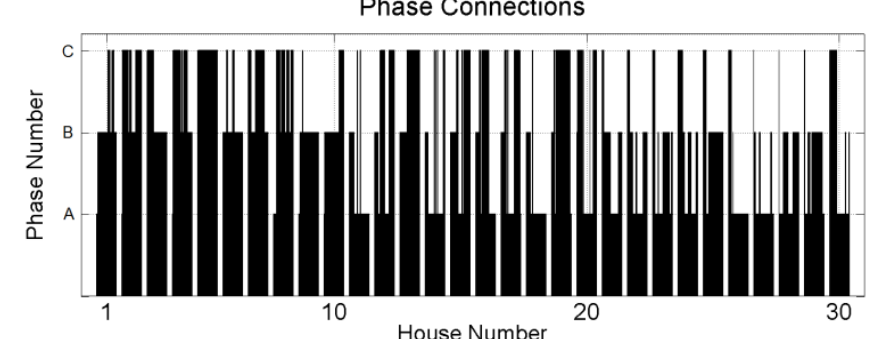

(f)

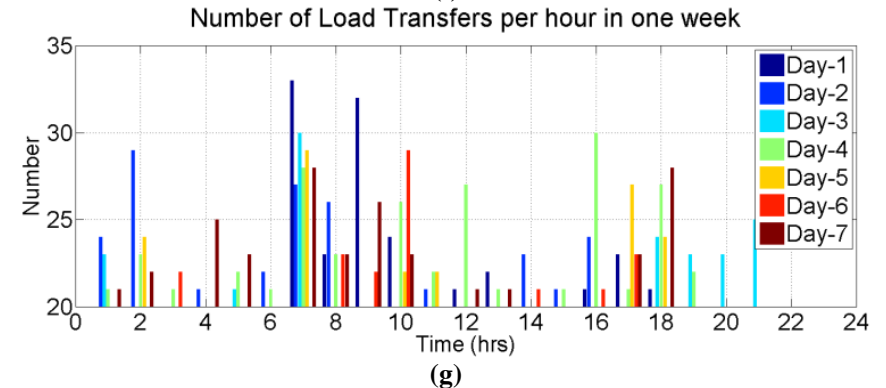

(g)
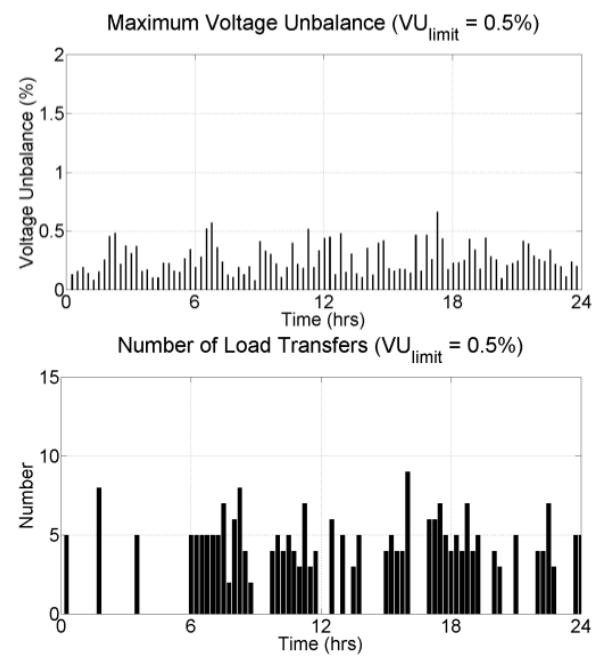

Fig. 6. Analysis results for the LT scheme based on highly-loaded to low-loaded control algorithm with deadband control with $0.5 \%, 1 \%$ and $1.5 \%$ level of acceptable VU: (Top row) maximum VU in the feeder after LTs, (Bottom row) Total number of LTs/switching event. 

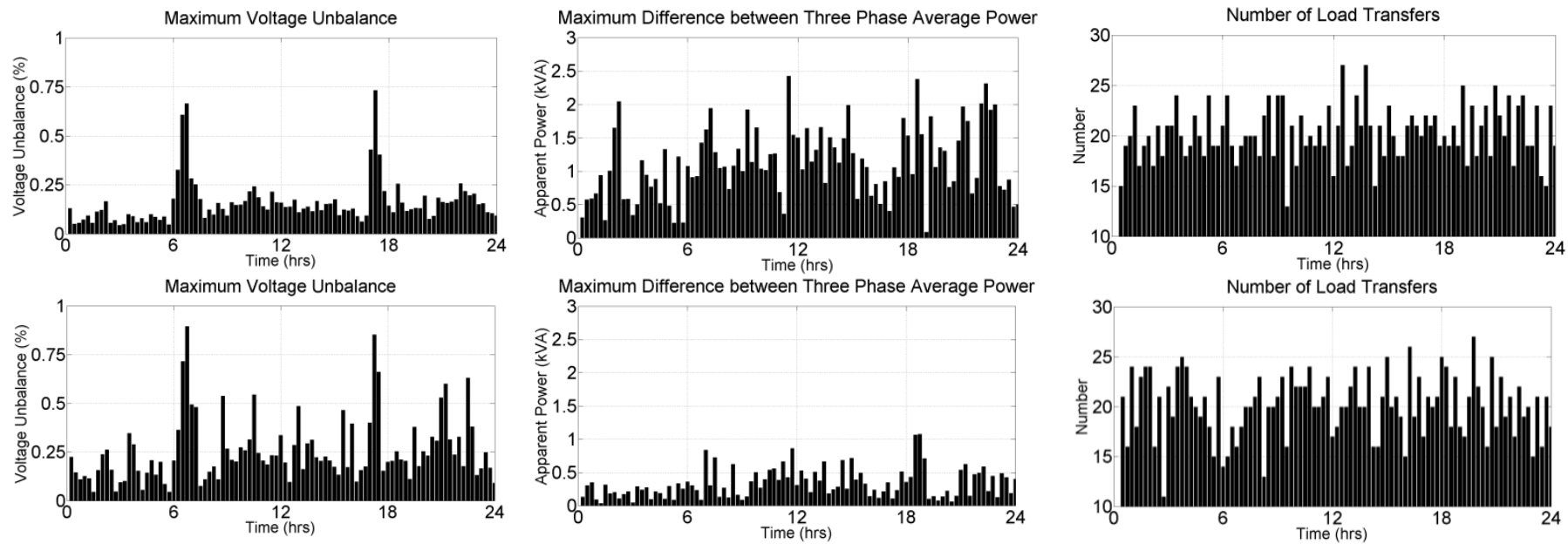

Maximum Difference between Three Phase Average Power
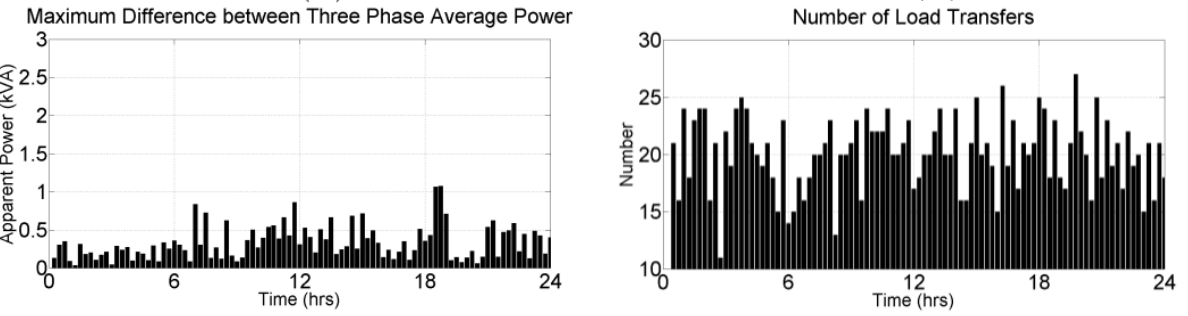

Fig. 7. Analysis results for the LT scheme using GA-based optimisation: (Top row) VU minimization case, (Bottom row) Both VU and power mismatch minimization case. (a)

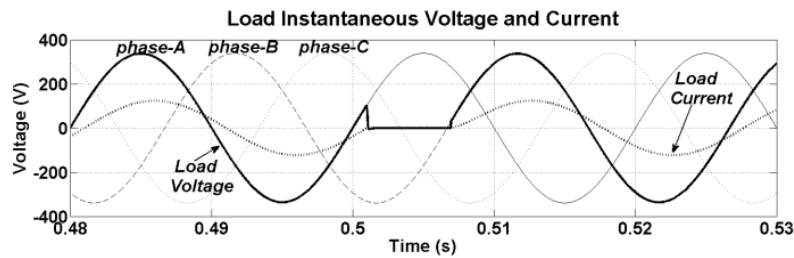

(b)

(c)

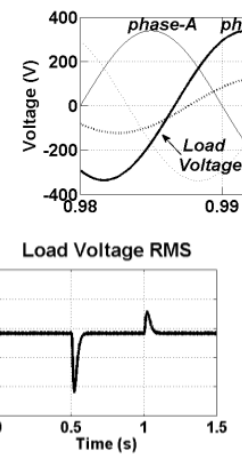

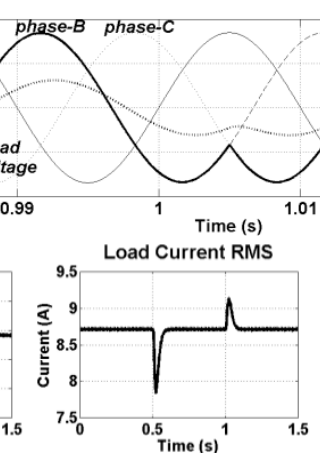

(d)

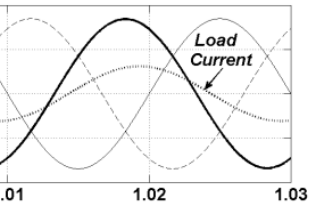

Load Active Power Demand

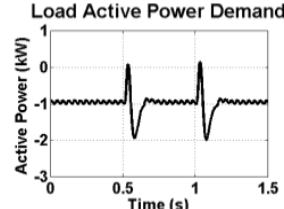

(e)
Fig. 8. Dynamic simulation results for an STS-based LT:

(a) Load instantanouse voltage for a LT at $t=0.5 \mathrm{~s}$ from Phase-A to B,

(b) Load instantanouse voltage for a LT at $t=1 \mathrm{~s}$ from Phase-B to C,

(c) Load RMS voltage,

(d) Load RMS current,

(e) Load active power in presence of a PV (negative load demand).

As the control command is received at $t=0.5 \mathrm{~s}$, the controller blocks the Phase-A Triac and de-blocks Phase-B Triac. However, the load current is not zero; therefore, the conducting thyristor still continues to pass the current until the current falls below the thyristor's holding current. This happens at $t=0.51 \mathrm{~s}$. STS starts to conduct at $t=0.57 \mathrm{~s}$, when the voltage of Phase-B is in its positive half cycle and its relevant thyristor is activated. This causes a slight drop in RMS voltage; however, it is within the acceptable region of the ITI curve.

At $t=1 \mathrm{~s}$, another LT command is received to transfer the load to Phase-C. At this time, the current is in its negative half cycle while Phase-C voltage is in its positive half cycle. Therefore, Phase-B Triac conducts the current until the current falls below the thyristor's holding current. This happens at $t=1.05 \mathrm{~s}$. Thereafter, the conducting Triac is turned off and Phase-C Triac starts to conduct simultaneously, as the voltage of Phase- $\mathrm{C}$ is in its negative half cycle and its relevant thyristor is activated. This results in a slight increase in RMS voltage; however, it is again within the acceptable region of the ITI curve.

As seen from Fig. 8(c), the RMS voltage variations, averaged over one cycle, in a LT transition period is below $10 \%$ and does not last more than $100 \mathrm{~ms}$; which falls within the acceptable region of the ITI curve.

The proposed LT algorithm should also work satisfactorily tributed generation units (e.g. PV or battery). For this, a residential load of $1 \mathrm{~kW}$ is considered, while its connected PV generates $2 \mathrm{~kW}$ - causing a negative load demand of $1 \mathrm{~kW}$. The PV unit, modelled based on the voltage-current characteristic of PV cells is assumed to be connected to the load through a single-phase inverter, as discussed in [32]. Fig. 8(e) shows the load active power demand while the abovementioned LTs are applied again. This figure shows that, even in the case of a reverse power flow, the voltage variations are within the acceptable regions of the ITI curve. The simulation results verify successful dynamic performance of the proposed LT for residential applications using the new connection strategy for the STS.

An intelligent dynamic residential load transfer scheme is proposed in this paper. Houses can be transferred between phases based on the commands from a central controller and with the help of a static transfer switch. The central controller utilizes the proposed highly-loaded to low-loaded phase transfer algorithm to define the candidate load(s) to be transferred, while keeping the number of load transfers to minimum. The load transfer scheme is discrete and operates on 15-min intervals. A deadband controller is developed to reduce the number of load transfers by allowing a small but acceptable level of voltage unbalance in the network. It can also utilize a GA-based optimization for voltage unbalance and power mismatch reduction among the three phases but in case of a reverse power flow, caused by single-phase dis-

\section{CONCLUSION}


will lead to much larger number of load transfers. The effectiveness of the proposed method is been verified through MATLAB analyses of a typical Australian LV distribution feeder using the load profile data available from smart meters. The analysis results for a 24-hr period showed how effectively the voltage unbalance is reduced by applying the developed load transfer scheme. Similarly, the results for a 7day period showed that a minimum of 2 and a maximum of 13 load transfers are applied for each house of the system under consideration to achieve minimum voltage unbalance along the feeder. The dynamic simulations of the static transfer switch demonstrates that the voltage disturbance during each load transfer falls within the acceptable region of the input voltage for the electrical appliances, based on the ITI curve.

The vector analysis method, introduced in this paper, can be developed into a selection algorithm to identify the LT candidate $\operatorname{load}(\mathrm{s})$. In addition, some future work can be carried out to investigate the dynamic performance of singlephase motors and inverter type loads and generators in residential premises in load transfer transition periods. Similarly, the dynamic interactions for the network consisting of several loads with PVs and several static transfer switches can be studied as another future work.

\section{REFERENCES}

[1] T. A. Short, Electric Power Distribution Handbook, CRC Press, 2004.

[2] A. Ghosh and G. Ledwich, Power Quality Enhancement using Custom Power Devices, Kluwer Academic, 2002.

[3] F. Shahnia, R. Majumder, A. Ghosh, G. Ledwich and F. Zare, "Voltage imbalance analysis in residential low voltage distribution networks with rooftop PVs," Electric Power Systems Research, Vol. 81, Issue 9, pp. 1805-1814, Sept. 2011.

[4] F. Shahnia, A. Ghosh, G. Ledwich and F. Zare, "Predicting voltage unbalance impacts of plug-in electric vehicles penetration in residential low voltage distribution networks," Electric Power Components and Systems, Vol. 41, Issue 16, pp. 1594-1616, Oct. 2013.

[5] F. Shahnia, A. Ghosh, G. Ledwich and F. Zare, "Voltage unbalance improvement in low voltage residential feeders with rooftop PVs using custom power devices," International Journal of Electrical Power \& Energy Systems, Vol. 55, pp. 362-377, Feb. 2014.

[6] R.J. Sarfi, M.M.A. Salama and A.Y. Chikhani, "A survey of the state of the art in distribution system reconfiguration for system loss reduction," Electric Power Systems Research, Vol. 31, Issue 1, pp. 61-70, 1994.

[7] C. Lueken, P.M.S. Carvalho and J. Apt, "Distribution grid reconfiguration reduces power losses and helps integrate renewables," Energy Policy, Vol. 48, pp. 260-273, Sept. 2012.

[8] S. Jazebi and B. Vahidi, "Reconfiguration of distribution networks to mitigate utilities power quality disturbances," Electric Power Systems Research, Vol. 91, pp. 9-17, Oct. 2012.

[9] B. Amanulla, S. Chakrabarti and S.N. Singh, "Reconfiguration of power distribution systems considering reliability and power loss," IEEE Trans. on Power Delivery, Vol. 27, No. 2, pp. 918-926, 2012.

[10] T.H. Chen and J.T. Cherng, "Optimal phase arrangement of distribution transformers connected to a primary feeder for system unbalance improvement and loss reduction using a genetic algorithm," IEEE Trans. on Power Systems, Vol. 15, No. 3, pp. 994-1000, Aug 2000.

[11] A. Ghosh, "Performance study of two different compensating devices in a custom power park," IEE Proc. Generation, Transmission and Distribution, Vol. 152, No. 4, pp. 521- 528, July 2005.

[12] A.K. Singh, G.K. Singh and R. Mitra, "Some observations on definitions of voltage unbalance," $39^{\text {th }}$ North American Power Symposium (NAPS), pp. 473-479, Sep./Oct. 2007.

[13] M.T. Bina and A. Kashefi, "Three-phase unbalance of distribution systems: Complementary analysis and experimental case study," Inter- national Journal of Electrical Power and Energy Systems, Vol. 33, Issue 4, pp. 817-826, May 2011.

[14] IEEE Recommended Practice for Monitoring Electric Power Quality, IEEE Standard 1159-1995.

[15] Planning Limits for Voltage Unbalance in the United Kingdom, The Electricity Council, Engineering recommendation P29, 1990.

[16] Electric Power Systems and Equipment-Voltage ratings (60 Hz), ANS Standard C84.1-1995.

[17] D.C. Garcia, A.L.F. Filho, M.A.G. Oliveira, O. A. Fernandes, F.A. do Nascimentoa, "Voltage unbalance numerical evaluation and minimization," Electric Power Systems Research, Vol. 79, pp. 1441-1445, 2009.

[18] H. Pezeshki and P. Wolfs, "Correlation based method for phase identification in a three phase LV distribution network," $22^{\text {nd }}$ Australasian Universities Power Engineering Conf. (AUPEC), pp.1-7, Sept. 2012.

[19] S.S.S.R. Depuru, W. Lingfeng, V. Devabhaktuni and N. Gudi, "Smart meters for power grid: Challenges, issues, advantages and status," $R e$ newable and Sustainable Energy Reviews, Vol. 15, Issue 6, pp. 27362742, Aug. 2011.

[20] J. Gao, Y. Xiao, J. Liu, W. Liang and C.L.P. Chen, "A survey of communication/networking in Smart Grids," Future Generation Computer Systems, Vol. 28, Issue 2, pp. 391-404, Feb. 2012.

[21] M.E. Kantarci and H.T. Mouftah, "Supply and load management for the smart distribution grid using wireless networks," IEEE Japan-Egypt Conference on Electronics, Communications and Computers (JECECC), pp.145-150, March 2012.

[22] D. Niyato, W. Ping and E. Hossain, "Reliability analysis and redundancy design of smart grid wireless communications system for demand side management," IEEE Wireless Communications, Vol. 19, No. 3, pp. 38-46, June 2012.

[23] Y. Shaoyong, A. Bryant, P. Mawby, X. Dawei, L. Ran and P. Tavner, "An industry-based survey of reliability in power electronic converters," IEEE Trans. on Industry Applications, Vol. 47, No. 3, pp. 14411451, May/June 2011.

[24] G.T. Heydt, R. Ayyanar and R. Thallam, "Power Acceptability," IEEE Power Engineering Review, Vol. 21, No. 9, pp. 12-15, Sept. 2001.

[25] S. Elphick and V. Smith, "The 230V CBEMA curve- preliminary studies," $20^{\text {th }}$ Australasian Universities Power Engineering Conference (AUPEC), pp. 1-6, Dec. 2010.

[26] A. Faruqui, D. Harris and R. Hledik, "Unlocking the $€ 53$ billion savings from smart meters in the EU: How increasing the adoption of dynamic tariffs could make or break the EU's smart grid investment, Energy Policy, Vol. 38, pp. 6222-6231, 2010.

[27] J. Zhu, Optimization of Power System Operation, Wiley, 2009.

[28] Olex Aerial Catalogue, (Available at http://www.olex.com.au/Australasia/2012/OLC12641 AerialCat.pdf)

[29] Western Power Distribution Construction Standards Handbook, Part 6Low Voltage Overhead, 2007. (Available at

http://www.westernpower.com.au/documents/networkcontractors/distri butioncon-

struc/we_n5115114_v2_distribution_construction_standards_handbook .pdf).

[30] Y. Li and P. Wolfs, "Hybrid Model for Residential Loads in a Distribution System With High PV Penetration," IEEE Trans. on Power Systems, Vol. 28, No. 3, pp. 3372-3379, Aug. 2013.

[31] W.H. Kersting, Distribution System Modeling and Analysis, CRC Press, 2012.

[32] F. Shahnia, R. Majumder, A. Ghosh, G. Ledwich and F. Zare, "Operation and control of a hybrid microgrid containing unbalanced and nonlinear loads," Electric Power Systems Research, Vol. 80, Issue 8, pp. 954-965, Aug. 2010. 
Page 11 